\title{
Recommendations for enhancing collaboration between the Canadian emergency department quality improvement and research communities
}

\author{
Lucas B. Chartier ${ }^{1,2}$ - Stuart L. Douglas ${ }^{3,4}$ - Davy Tawadrous ${ }^{1,2} \cdot$ Antonia S. Stang $^{5,6} \cdot$ Samuel Vaillancourt $^{2,7}$. \\ Laila Nasser $^{8} \cdot$ Carmen Hrymak $^{9,10} \cdot$ Lisa Calder $^{11,12}$ - Jeffrey J. Perry ${ }^{11,12} \cdot$ Andrew McRae $^{6,13}$
}

Received: 18 December 2020 / Accepted: 24 December 2020 / Published online: 9 February 2021

(C) The Author(s), under exclusive licence to Canadian Association of Emergency Physicians (CAEP)/ Association Canadienne de Médecine d'Urgence (ACMU) 2021

\begin{abstract}
Objectives While quality improvement (QI) and clinical research embody two distinct scientific approaches, they have the same ultimate goal - to improve health and patient care outcomes. By leveraging their respective strengths there is a higher likelihood of achieving and sustaining health improvements. Our objective was to create recommendations to enhance the collaboration of the Canadian emergency medicine QI and clinical research communities.

Methods An expert panel of eight ED clinicians with diverse QI and clinical research expertise drafted a list of recommendations based on their professional expertise and a scoping review of the literature. These recommendations were refined through consultation with national stakeholders and reviewed at the 2020 CAEP Virtual Academic Symposium, where feedback was received through several virtual platforms.

Results The final six recommendations include that all emergency medicine providers should: (1) understand the role and application of both clinical research and QI science; that academic emergency medicine physicians should: (2) contribute to both local adoption and broad dissemination of project findings, (3) leverage QI methodologies in research projects to improve knowledge translation, and (4) ensure that project outcomes prioritize patient care; and that academic leaders should: (5) enhance the infrastructure for oversight of research and QI projects, and (6) encourage collaboration between researchers and QI experts by ensuring that academic and operational infrastructures align and support both.

Conclusion Six recommendations are presented to help the Canadian emergency medicine community achieve greater collaboration between researchers and QI experts with the ultimate goal of improving patient care outcomes.
\end{abstract}

Keywords Emergency medicine $\cdot$ Quality improvement $\cdot$ Research methods $\cdot$ Patient oriented

\section{Résumé}

Objectifs Bien que l'amélioration de la qualité (AQ) et la recherche clinique représentent deux approches scientifiques distinctes, elles ont le même but ultime: améliorer la santé et les résultats des soins aux patients. En tirant profit de leurs atouts respectifs, les chances d'obtenir et de soutenir des améliorations de santé sont plus élevées. Notre objectif était de créer des recommandations pour renforcer la collaboration entre les communautés d'AQ et de recherche clinique en médecine d'urgence canadienne.

Méthodes Un groupe d'experts de huit cliniciens des services d'urgence dotés d'une expertise diversifiée en matière d'AQ et de recherche clinique a rédigé une liste de recommandations basées sur leur expertise professionnelle et un examen de la revue de littérature. Ces recommandations ont été affinées en consultation avec les parties prenantes nationales et examinées lors du Symposium académique virtuel de ACMU 2020, où des commentaires ont été reçus via plusieurs plateformes virtuelles. Résultats Les six recommandations finales incluent que tous les prestataires des services de médecine d'urgence devraient: (1) comprendre le rôle et l'application de la recherche clinique et de la science de l'AQ; que les médecins universitaires en

Lucas B. Chartier

lucas.chartier@uhn.ca

Extended author information available on the last page of the article 
médecine d'urgence devraient: (2) contribuer à la fois à l'adoption locale et à la diffusion large des résultats de projets, (3) tirer parti des méthodologies d'AQ dans les projets de recherche afin d'améliorer l'application des connaissances, et (4) veiller à ce que les résultats de projet donnent la priorité aux soins aux patients; et que les dirigeants universitaires devraient: (5) améliorer l'infrastructure de surveillance des projets de recherche et d'AQ, et (6) encourager la collaboration entre les chercheurs et les experts en AQ en assurant que les infrastructures universitaires et opérationnelles les alignent et les soutiennent. Conclusion Six recommandations sont présentées pour aider la communauté de la médecine d'urgence canadienne à parvenir à une plus grande collaboration entre les chercheurs et les experts en AQ dans le but ultime d'améliorer les résultats des soins aux patients.

\section{Background}

The objective of clinical and health systems research (herein referred to as 'research') is typically to advance our understanding of human health and/or to create new knowledge. The objective of quality improvement (QI) is to iteratively refine a system to improve a pre-determined outcome. Its methodologies are increasingly recognized as having the potential to contribute to the design, conduct, and analysis of research programs [1]. In some cases, this mixed-methods approach may more efficiently achieve measurable improved patient and process of care outcomes [2].

The Canadian emergency medicine (EM) community is uniquely positioned to operationalize the benefits of enhanced collaboration between research and QI [1]. Opportunities for enhanced and mutually beneficial partnerships exist at many levels, including EM providers, academic EM physicians (i.e. both researchers and QI experts), and academic leaders (e.g., EM Chairs, emergency department (ED) Chiefs, and ED Research or QI Directors). These constituency groups all have an intrinsic responsibility to the advancement of the quality of care, with the ultimate objective of improved patient outcomes.

Through a scoping review of the literature and a national stakeholder engagement process, we sought to identify concrete ways through which collaboration between researchers and QI experts in the EM community can be facilitated. Herein, we provide the recommendations stemming from this process and we describe complementary methodologies to maximize project impact.

\section{Methods}

\section{Expert panel process}

We formed an expert panel that included seven staff emergency physicians and one EM resident, affiliated with six different Canadian medical schools. Panel members were selected based on expertise in QI, patient safety and research, as well as diversity of professional experience and geographic representation. Panel members' roles include heads of departmental quality and/or research programs, national leadership roles, and front-line clinicians.

The panel developed a draft list of recommendations based on a scoping review of the literature, as well as their professional experience and academic expertise. The panel then iteratively refined these recommendations through a series of phone discussions facilitated by the co-chairs (LBC and SLD) over the course of ten months.

\section{Stakeholder engagement}

The expert panel sought feedback from 14 members of the Canadian EM community, including seven identified as QI experts and nine identified as researchers (two fulfilled both descriptions). This group included experts, thought and academic leaders, peer-reviewed journal editors, and front-line emergency medicine clinicians. Based on their feedback, recommendations were refined with evidence referenced where possible to ensure validity.

\section{Public comment}

As a result of the COVID-19 pandemic, the Canadian Association of Emergency Physicians (CAEP) 2020 Academic Symposium where the draft recommendations were meant to be discussed in person was canceled. Instead, the recommendations were shared with a group of stakeholders for feedback and improvement, including members of the CAEP QIPS Committee and research community. This iterated product was then presented at the CAEP Virtual Symposium on October 1st, 2020, for final validation and refinement, using three methods for feedback: real-time chat, PollEverywhere ${ }^{\circledR}$ (San Francisco, CA, USA) comments, as well as a post-Symposium survey of attendees.

\section{Recommendations}

To enhance collaboration between QI experts and researchers, we defined the following recommendations aimed at EM providers, academic EM physicians, and academic leaders (Table 1). 
Table 1 Summary of recommendations

\begin{tabular}{ll}
\hline Target audience & Recommendations \\
\hline Emergency medicine providers & $\begin{array}{l}\text { 1. All emergency medicine providers should understand the role and application of both clinical } \\
\text { research and quality improvement science }\end{array}$ \\
Academic emergency medicine physicians & $\begin{array}{l}\text { 2. Academic emergency medicine physicians should contribute to both local adoption and broad } \\
\text { dissemination of project findings }\end{array}$ \\
3. Quality improvement methodology should be leveraged by researchers to improve the knowledge \\
translation of study findings \\
4. Researchers and quality improvement experts should ensure that their respective project outcomes \\
prioritize patient care \\
5. Academic leaders should strive to enhance the infrastructure for oversight of research and quality \\
improvement projects \\
6. Academic leaders should encourage collaboration between researchers and quality improvement \\
experts by ensuring that academic and operational infrastructures align and support both
\end{tabular}

\section{Emergency medicine providers}

\section{Recommendation 1. All emergency medicine providers should understand the role and application of both clinical research and quality improvement science}

A basic understanding of both clinical research and QI methods will enable EM providers to adopt the tools most suited for addressing the clinical challenges they face. Although there are differences in purpose and methods between QI and research, the underlying goal of both is usually to improve health care and patient outcomes. Both rely on stakeholder engagement, creative and adaptive designs, meticulous and systematic measurement, effective data management, and thoughtful dissemination of results [1]. Without research there is no new knowledge to implement, and the creation of new knowledge in isolation will not result in improved patient care.

Undergraduate and postgraduate medical education curricula for research have matured over decades, but Canadian postgraduate QI curricula lack similar structure and opportunities [3]. There must be a joint effort by respective experts to ensure that enhanced academic curricula cover both research and QI methodologies. Curricula may include encouraging a minimum research and QI literacy requirement for all trainees (supported by the inclusion of competencies reflecting patient safety and quality improvement in CanMEDS 2015 [4]), providing comprehensive project and mentorship opportunities, and ensuring that trainees willing to engage in further training in either or both streams are appropriately supported [5]. Training, mentorship and professional development opportunities are needed for practicing physicians in both research and QI methodologies. To facilitate access to resources for enhancing QI knowledge, CAEP has developed a QIPS Resource webpage [6].

\section{Academic emergency medicine physicians}

\section{Recommendation 2. Academic emergency medicine physicians should contribute to both local adoption and broad dissemination of project findings}

The ideal outcome for research should be both dissemination of new knowledge globally and the successful adoption of effective practices locally; the latter sometimes being particularly challenging to successfully implement and sustain. Conversely, rigorous health care QI programs exist and are often carried out with the primary goal of improving local care. However, their impact can be impaired without effective academic dissemination. This results in repetition of projects between locales, resulting in unnecessary waste. This is partly due to the overlap of QI projects with operational tasks and change management approaches, as well as project completion by leads not traditionally driven by scholarly dissemination [7]. On the other hand, research success is often measured by its ability to contribute to practice guidelines or scientific statements, and dissemination of results in peer-reviewed journals. The corollary to this strength is the historically lower emphasis on translating these findings into practical and sustainable local impact [8].

A new way forward that allows for broad dissemination and local adoption of all projects is needed. Shared learning has proven beneficial with Quality Improvement Collaboratives (QICs), where multiple sites benefit from the exchange of evidence-based practices despite variability between sites. This collaborative approach may prove to be a useful method for implementing research findings into multiple healthcare settings [9, 10]. The Network of Canadian Emergency Researchers (NCER) is another potential platform for QI and research teams to collaborate and share their work. 


\section{Recommendation 3. Quality improvement methodology should be leveraged by researchers to improve the knowledge translation of study findings}

Traditionally, peer-reviewed dissemination (i.e. end-of-grant knowledge translation) has been the vehicle for knowledge spread [10]. This possibly contributes to a knowledge translation 'gap', whereby new knowledge is not incorporated into clinical practice in a timely fashion [11]. Clinical researchers have increasingly adopted a broader set of approaches to close this knowledge translation gap, leading to greater realized impact. Inclusion of QI methodologies throughout the life cycle of projects, otherwise known as integrated knowledge translation, can further augment such efforts, but they should be considered at the earliest stages of protocol development (Table 2). This could include, for example, earlier meaningful engagement of front-line providers and/or patients to appraise the relevance of project hypotheses and findings; use of alternative methodologies such as process flow maps, cause and effect analyses, or Pareto charts [12]; and iteratively refining study interventions to enhance local adoption and sustainability. Given that multiple barriers to implementation exist even with the presence of clear clinical practice guidelines, collaboration between researchers and QI experts can help ensure the production of quality evidence and its translation into sustainable process improvements $[8,9]$.

\section{Recommendation 4. Researchers and quality improvement experts should ensure that their respective project outcomes prioritize patient care}

Improving patient outcomes is an objective of most EM research programs. Although the broad strategic vision and funding of research programs are influenced by various leaders and organizations outside of EM, researchers have a role in ensuring that the focus remains centered on patients. As EM research diversifies, it is incumbent on researchers and QI experts to seek and highlight links between their projects and specific patient-oriented outcomes.

Further, research should strive to ensure that findings are broadly applicable, and QI experts should promote this through the development of a thoughtful family of measures, the inclusion of end-users and providers in measure selection, and the performance of usability testing to ensure that the measures selected reflect the intended outcomes.

Table 2 Quality improvement methodology relevant to various steps of research studies

\begin{tabular}{|c|c|}
\hline Research component & Related quality improvement methodology \\
\hline Development of study question & $\begin{array}{l}\text { Patient co-design to ensure meaning/relevance to patients } \\
\text { Stakeholder analysis for adequate depth of engagement of various groups (e.g., patients/ } \\
\text { caregivers, front-line interprofessional providers, departmental leaders, executive sponsors } \\
\text { at upper leadership level) } \\
\text { Identification of a problem statement to guide planning } \\
\text { Building a burning platform to ensure local leadership commitment, stakeholder engage- } \\
\text { ment and front-line buy-in (e.g., champions) } \\
\text { Consideration of hybrid designs (e.g., quasi-experimental, interrupted time-series), espe- } \\
\text { cially when randomization impossible }\end{array}$ \\
\hline Protocol development and intervention(s) selection & $\begin{array}{l}\text { Ishikawa (fishbone) diagram to identify all relevant causal elements for the interprofessional } \\
\text { team } \\
\text { Process mapping of complex systems to illustrate optimal flow and timing of } \\
\text { intervention(s), as well as feasibility within local context (e.g., early identification of } \\
\text { system barriers) } \\
\text { Effort-Impact diagram and/or Driver diagram of change ideas and drivers towards the over- } \\
\text { all aim to select the highest-yield approaches } \\
\text { Rapid-cycle iteration (i.e. Plan-Do-Study-Act cycles) and refinement of intervention(s) } \\
\text { through pilot testing to ensure their highest-yield impact once implemented in a defined } \\
\text { study protocol }\end{array}$ \\
\hline Evaluation and analytical plan & $\begin{array}{l}\text { Repeated data sampling to assess progress toward aims, detect change, and improve effi- } \\
\text { ciency } \\
\text { Run chart and/or Statistical process control (SPC) chart to identify special cause variation } \\
\text { (i.e. signal in the noise of expected process variation) } \\
\text { Effectiveness-implementation hybrid designs }\end{array}$ \\
\hline Scale, spread and sustainability planning & $\begin{array}{l}\text { Consideration of contexts (micro, meso and macro-levels) to ensure success and replicabil- } \\
\text { ity } \\
\text { Use of highly adoptable improvement model for long-term sustainability } \\
\text { Use of models for spread }\end{array}$ \\
\hline
\end{tabular}

N.B. The Quality Improvement Primer series in the Canadian Journal of Emergency Medicine gives further information on a number of these topics $[12,20,21]$ 
Valuing the effectiveness of study interventions on patients and ensuring that knowledge benefits patients beyond the study period requires a multipronged approach of rigorous trials (and errors) and can be supported by QI methodology [13]. Similarly, QI projects must ensure that the proposed cycles of change are evidence supported.

\section{Academic leaders}

\section{Recommendation 5. Academic leaders should strive to enhance the infrastructure for oversight of research and quality improvement projects}

As we progress toward a data-rich healthcare environment, the differentiation between QI and research is sometimes hard to make [14]. New pathways are needed to ensure risks to patients are minimized both from research but also from unaddressed correctable quality gaps. Canadian research abides by the Tri-Council Policy Statement for the Ethical Conduct for Research Involving Humans [15]. These standards are written specifically for situations where participants are subject to added risks beyond changes to routine care, and they are typically enforced through local Research Ethics Boards (REBs). Many organizations require QI projects to be reviewed through the same pathway. This process, as currently designed, may prolong patients' exposure to preventable harm by making low-risk projects unworkable, limit the ability to use iterative methods, and increase the length of time required for all REB reviews. Additionally, low-risk research initiatives may warrant proportionate-risk REB review.

A more discerning project oversight process could be used constructively by both researchers and QI experts to ensure appropriate reviews for all project types and enable the appropriate level of oversight for proposed interventions. This process could include stratification of review processes: low-risk projects being required to meet the standards of routine health care delivery, moderate-risk projects subject to advisory group review, and high-risk projects (e.g., with added risks to patients, or those involving external funding, conflicts of interests or traditional research components) required to meet typical Tri-Council requirements [16]. This would ensure that all studies have rigorous oversight where added patient risk is involved, but also encourage low-risk projects without significant change to routine health care delivery.

One tool that may be included in this process' decisionmaking matrix is the ARECCI (A pRoject Ethics Community Consensus Initiative) tool. It is a screening tool that provides decision-support guidance to teams for alternative methodology projects involving people or personal health information, based on the level of risk for the participants [17]. Created for use in Alberta, Canada, it provides a template which clarifies when REB oversight is required for specific projects.

\section{Recommendation 6. Academic leaders should encourage collaboration between researchers and quality improvement experts by ensuring that academic and operational infrastructures align and support both}

It behooves universities, health care organizations and EM departments to build successful and sustainable academic programs. These require support from all levels of leadership (i.e. departmental, organizational and university) on: frameworks aligning research and QI, explicit pathways for both to support academic promotion, and adequate funding strategies.

One strategy is for academic leaders to align processes to leverage engagement of researchers and QI experts. This could include the creation of multi-modal academic networks (i.e. research and QI combined), encouragement of representation of relevant experts on each other's working groups, and even incentives and rewards for cross-collaboration, such as time in lieu of academic pursuits or points systems. As collaborative academic endeavors mature in the coming years, efforts should focus on developing local frameworks to appropriately match a clinical hypothesis or problem to the optimal methodology: research, QI, or hybrid research-QI. These local frameworks should also understand and acknowledge the differences between QI and research to maintain the integrity of both while enabling integration. Ultimately, this will help integrate academic endeavors with operational priorities.

Another strategy is to link academic leadership performance with the diversification of an academic unit's portfolio. This goal is in line with salary benefits for hospital executives that are often tied to success in the organization's Quality Improvement Plan, and it can be achieved in part by the development of sustainable academic funding and collaboration for data management.

Securing funding from innovative and diversified sources is essential to create a sustainable and robust research program, including non-traditional sources (e.g., philanthropy, operational funds, etc.) so that projects of local importance can be supported [18]. QI expands possible funding sources and may improve process efficiency and cost. For example, in a national survey of Canadian EM QIPS leads, $18 \%$ indicated their contribution to the departmental funding through external peer-reviewed QIPS grants [19].

\section{Conclusion}

We described considerations for enhanced collaboration between QI experts and researchers, with a goal of forming a joint team with diverse expertise rather than perpetuating 
the silos that may have inadvertently developed over time. All members are aligned in their ultimate goal of improving patient outcomes, with the differences in methodology and implementation being a strength when pooled. These recommendations describe how collaboration can be mutually beneficial, with tangible action items at the levels of EM providers, academic EM physicians and academic leaders.

Acknowledgements The authors acknowledge the input of these 14 experts who helped refine our thinking and these recommendations (alphabetically): Rob Brison, Teresa Chan, Adam Dukelow, Stephen Freedman, Eddy Lang, Murdoch Leeies, Matthew Mercuri, Shawn Mondoux, Laurie Morrison, Olivia Ostrow, Naveen Poonai, Michael Schull, Kaveh Shojania and Jennifer Thull-Freedman.

\section{Compliance with ethical standards}

Conflict of interest The authors declare no conflict of interests.

\section{References}

1. Margolis P, Provost LP, Schoettker PJ, et al. Quality improvement, clinical research, and quality improvement research-opportunities for integration. Pediatr Clin North Am. 2009;56:831-41. https:// doi.org/10.1016/j.pcl.2009.05.008.

2. Horwitz LI, Kuznetsova M, Jones SA. Creating a learning health system through rapid-cycle, randomized testing. N Engl J Med. 2019;381:1175-9. https://doi.org/10.1056/NEJMsb1900856.

3. Trivedi SV, Hartmann RJ, Hall JN, et al. Residents' perspective of quality improvement and patient safety education in Canadian emergency medicine residency programs. CJEM. 2020. https:// doi.org/10.1017/cem.2019.465.

4. Frank JR, Snell L, Sherbino J. CanMEDS 2015. Ottawa: Physician Competency Framework; 2015.

5. Chartier LB, Mondoux SE, Stang AS, et al. How do emergency departments and emergency leaders catalyze positive change through quality improvement collaborations? Can J Emerg Med. 2019;21:542-9. https://doi.org/10.1017/cem.2019.25.

6. QIPS Resources-CAEP. https://caep.ca/qips-resources/. Accessed 31 Jan 2020.

7. Portela MC, Pronovost PJ, Woodcock T, et al. How to study improvement interventions: a brief overview of possible study types. BMJ Qual Saf. 2015;24:325-36. https://doi.org/10.1136/ bmjqs-2014-003620.

8. Grimshaw J, Eccles M, Tetroe J. Implementing clinical guidelines: current evidence and future implications. J Contin Educ Health Prof. 2004;24(1):S31-7. https://doi.org/10.1002/chp.1340240506 PMID: 15712775
9. Kajermo KN, Boström A, Thompson DS, et al. The BARRIERS scale-the barriers to research utilization scale: A systematic review. Implementation Sci. 2010;5:32. https://doi. org/10.1186/1748-5908-5-32

10. Wensing M, Grol R. Knowledge translation in health: how implementation science could contribute more. BMC Med. 2019;17:88. https://doi.org/10.1186/s12916-019-1322-9.

11. Sung NS, Crowley WF, Genel M, et al. Central challenges facing the national clinical research enterprise. J Am Med Assoc. 2003;289:1278-87. https://doi.org/10.1001/jama.289.10.1278.

12. Chartier LB, Cheng AHY, Stang AS, et al. Quality improvement primer part 1: preparing for a quality improvement project in the emergency department. Can J Emerg Med. 2018;20:104-11. https ://doi.org/10.1017/cem.2017.361.

13. Dixon-Woods M. How to improve healthcare improvement-an essay by Mary Dixon-Woods. BMJ. 2019. https://doi.org/10.1136/ bmj.15514.

14. Faden RR, Kass NE, Goodman SN, et al. An ethics framework for a learning health care system: a departure from traditional research ethics and clinical ethics. Hastings Cent Rep. 2013;43:S16-27. https://doi.org/10.1002/hast.134.

15. Canadian Institutes of Health Research, Natural Sciences and Engineering Research Council of Canada, and Social Sciences and Humanities Research Council of Canada. Tri-Council Policy Statement: Ethical Conduct for Research Involving Humans. 2014. http://www.pre.ethics.gc.ca/eng/index/. Accessed 6 Jun 2019.

16. Baily MA, Bottrell M, Lynn J, et al. The ethics of using QI methods to improve health care quality and safety. Hastings Cent Rep. 2006;36:S1-40. http://www.ncbi.nlm.nih.gov/pubmed/16898359. Accessed 27 Jul 2019.

17. Hagen B, O'Beirne M, Desai S, et al. Innovations in the ethical review of health-related quality improvement and research: The Alberta Research Ethics Community Consensus Initiative (ARECCI). Healthc Policy 2007;2:e164-77. http://www.ncbi. nlm.nih.gov/pubmed/19305726. Accessed 6 Dec 2019.

18. Campbell EG. The future of research funding in academic medicine. N Engl J Med. 2009;360:1482-3. https://doi.org/10.1056/ NEJMp0900132.

19. Kwok ESH, Perry JJ, Mondoux S, et al. An environmental scan of quality improvement and patient safety activities in emergency medicine in Canada. CJEM. 2019;21:535-41. https://doi. org/10.1017/cem.2019.16.

20. Chartier LB, Stang AS, Vaillancourt S, et al. Quality improvement primer part 2: executing a quality improvement project in the emergency department. Can J Emerg Med. 2018;20:532-8. https://doi.org/10.1017/cem.2017.393.

21. Chartier LB, Vaillancourt S, Cheng AHY, et al. Quality improvement primer part 3: evaluating and sustaining a quality improvement project in the emergency department. Can J Emerg Med. 2019;21:261-8. https://doi.org/10.1017/cem.2018.380.

\section{Authors and Affiliations}

\section{Lucas B. Chartier ${ }^{1,2}$ (1) Stuart L. Douglas ${ }^{3,4} \cdot$ Davy Tawadrous $^{1,2} \cdot$ Antonia S. Stang $^{5,6}$. Samuel Vaillancourt ${ }^{2,7}$. Laila Nasser $^{8}$. Carmen Hrymak ${ }^{9,10} \cdot$ Lisa Calder $^{11,12}$. Jeffrey J. Perry ${ }^{11,12} \cdot$ Andrew McRae $^{6,13}$}

1 Department of Emergency Medicine, University Health Network, 200 Elizabeth St., RFE-GS-480, Toronto, ON M5G 2C4, Canada

2 Division of Emergency Medicine, Department of Medicine, University of Toronto, Toronto, ON, Canada
3 Department of Critical Care Medicine, Queen's University, Kingston, ON, Canada

4 Department of Emergency Medicine, Kingston Health Sciences Center, Kingston, ON, Canada 
5 Department of Pediatrics, Cumming School of Medicine, University of Calgary, Calgary, AB, Canada

6 Department of Emergency Medicine, Cumming School of Medicine, University of Calgary, Calgary, AB, Canada

7 Department of Emergency Medicine, St. Michael's Hospital, Unity Health Toronto, Toronto, ON, Canada

8 Division of Emergency Medicine, McMaster University, Hamilton, ON, Canada

9 Department of Emergency Medicine, Section of Critical Care, Rady Faculty of Health Sciences, University of Manitoba, Winnipeg, MB, Canada
10 Department of Internal Medicine, Section of Critical Care, Rady Faculty of Health Sciences, University of Manitoba, Winnipeg, MB, Canada

11 Department of Emergency Medicine, University of Ottawa, Ottawa, ON, Canada

12 Ottawa Hospital Research Institute, Ottawa, ON, Canada

13 Department of Community Health Sciences, Cumming School of Medicine, University of Calgary, Calgary, AB, Canada 